\title{
The Classification of Solvents by Combining Classical QSPR \\ Methodology with Principal Component Analysis
}

Alan R. Katritzky, Dan C. Fara, Minati Kuanar, Evrim Hur, Mati Karelson

Table SM 8. Loadings of the first five principal components of $(703 \times 100)$ matrix

\begin{tabular}{|c|c|c|c|c|c|c|}
\hline \multirow{2}{*}{$\begin{array}{l}\text { Groups } \\
\text { I }\end{array}$} & \multirow{2}{*}{$\begin{array}{l}\text { Scales } \\
\text { SPS3 / BCo }\end{array}$} & PC1 & \multirow{2}{*}{$\begin{aligned} \text { PC3 } \\
0.39\end{aligned}$} & PC4 & \multicolumn{2}{|c|}{ PC5 } \\
\hline & & 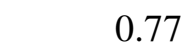 & & 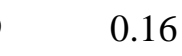 & -0.16 & 0.30 \\
\hline & SPS5 / BPe & 0.75 & 0.39 & -0.10 & -0.11 & 0.20 \\
\hline & SPS6 / Co & 0.78 & 0.35 & 0.07 & -0.03 & 0.17 \\
\hline & SPS8 / DCo & 0.69 & 0.44 & 0.10 & 0.05 & 0.34 \\
\hline & SPS11 / E ${ }_{\text {MLCT }}^{*}$ & 0.76 & 0.26 & -0.13 & -0.50 & -0.06 \\
\hline & SPS18 / G & 0.47 & 0.36 & -0.20 & -0.39 & -0.28 \\
\hline & SPS20 / K & 0.73 & 0.43 & 0.08 & -0.19 & 0.32 \\
\hline & SPS21 / NCo & 0.75 & 0.39 & -0.11 & -0.11 & 0.20 \\
\hline & SPS22 / Ov & 0.80 & 0.36 & 0.06 & -0.21 & 0.23 \\
\hline & $\mathrm{SPS} 23 / \mathrm{P}_{\mathrm{s}}$ & 0.79 & 0.18 & 0.37 & 0.05 & -0.01 \\
\hline & SPS24 / $\mathrm{P}_{\mathrm{y}}$ & 0.75 & 0.16 & 0.12 & -0.40 & 0.07 \\
\hline & SPS28 / $\mathrm{SPP}^{\mathrm{N}}$ & 0.74 & 0.05 & 0.35 & -0.22 & 0.08 \\
\hline & $\operatorname{SPS} 32 / \pi^{*}$ & 0.63 & 0.39 & 0.49 & -0.20 & -0.10 \\
\hline & SPS33 / $\pi_{\text {azo }}^{*}$ & 0.89 & -0.08 & 0.10 & 0.13 & -0.04 \\
\hline & $\mathrm{SPS} 36 / \int_{\mathrm{H}}^{\mathrm{P}-\mathrm{NO} 2}$ & 0.63 & 0.42 & -0.04 & 0.21 & 0.17 \\
\hline & $\operatorname{SPS} 39 / \Delta v_{\mathrm{A}}$ & 0.71 & 0.55 & 0.00 & -0.03 & -0.13 \\
\hline & SPS44 / $\lambda_{\mathrm{F}}{ }^{\mathrm{MHN} 12}$ & 0.69 & 0.28 & -0.19 & -0.10 & 0.30 \\
\hline & SPS $45 / \pi_{1^{*}}$ & 0.75 & 0.40 & 0.28 & -0.15 & -0.10 \\
\hline & SPS70 / E & 0.78 & -0.06 & 0.10 & 0.05 & 0.34 \\
\hline & SPS92 / d & 0.58 & -0.18 & -0.02 & -0.33 & 0.01 \\
\hline & SPS95 / J & 0.77 & -0.23 & 0.20 & -0.28 & 0.16 \\
\hline & SPS100 / N & 0.75 & -0.24 & 0.07 & -0.29 & 0.27 \\
\hline & SPS101 / P' & 0.88 & 0.10 & 0.13 & -0.01 & 0.07 \\
\hline & SPS102 / q- & 0.46 & -0.17 & 0.25 & -0.20 & 0.30 \\
\hline & SPS103 / q+ & 0.61 & -0.05 & 0.07 & 0.33 & 0.22 \\
\hline & SPS105 / S' & 0.77 & 0.19 & 0.19 & -0.36 & 0.26 \\
\hline & SPS107 / Xd ${ }^{R}$ & 0.47 & 0.65 & -0.03 & 0.35 & -0.24 \\
\hline & SPS110 / X & 0.58 & 0.52 & 0.02 & 0.32 & -0.17 \\
\hline & $\operatorname{SPS} 113 / \Delta \mathrm{G}_{6 \AA}$ & 0.64 & -0.16 & 0.39 & 0.20 & -0.05 \\
\hline & SPS119/ $\Theta\left(\epsilon_{B}\right)$ & 0.87 & 0.06 & 0.13 & -0.28 & 0.11 \\
\hline
\end{tabular}




\begin{tabular}{|c|c|c|c|c|c|c|}
\hline & SPS126 / Y & 0.84 & -0.08 & 0.04 & -0.24 & -0.02 \\
\hline & Min & 0.46 & -0.24 & -0.20 & -0.50 & -0.28 \\
\hline & Max & 0.89 & 0.65 & 0.49 & 0.35 & 0.34 \\
\hline \multirow[t]{20}{*}{ II } & SPS1 / AN & 0.72 & -0.18 & -0.21 & 0.51 & 0.00 \\
\hline & SPS4 / B & 0.31 & -0.15 & -0.35 & -0.42 & -0.46 \\
\hline & SPS13 / $\mathrm{E}_{\mathrm{B}}{ }^{\mathrm{N}}$ & 0.77 & -0.25 & -0.27 & 0.34 & -0.11 \\
\hline & SPS14 / $\mathrm{E}_{\mathrm{CT}(\mathrm{A})}$ & 0.66 & -0.39 & -0.25 & 0.33 & -0.07 \\
\hline & $\mathrm{SPS} 15$ / $\mathrm{E}_{\mathrm{T}}(30)$ & 0.87 & -0.29 & -0.08 & 0.16 & 0.03 \\
\hline & $\mathrm{SPS} 16$ / $\mathrm{E}_{\mathrm{T}}(\mathrm{N})$ & 0.85 & -0.30 & -0.16 & 0.14 & -0.04 \\
\hline & SPS17 / $\mathrm{E}_{\mathrm{T}}^{\mathrm{SO}}$ & 0.79 & -0.22 & -0.22 & 0.44 & 0.08 \\
\hline & SPS26 / SA & 0.65 & -0.32 & -0.36 & 0.41 & -0.17 \\
\hline & SPS29 / Z & 0.75 & -0.38 & -0.21 & 0.31 & -0.04 \\
\hline & SPS30 / $\alpha$ & 0.57 & -0.44 & -0.27 & 0.52 & -0.07 \\
\hline & SPS66 / Ф & 0.51 & -0.01 & -0.49 & -0.10 & 0.41 \\
\hline & SPS88 / A & 0.83 & -0.15 & -0.29 & 0.35 & -0.04 \\
\hline & SPS94 / E & 0.62 & -0.41 & -0.36 & 0.40 & -0.12 \\
\hline & SPS96 / Log K & 0.58 & -0.36 & -0.18 & 0.43 & 0.13 \\
\hline & SPS104 / S & 0.77 & 0.00 & -0.32 & 0.05 & -0.35 \\
\hline & SPS114 / $\Delta \mathrm{H}^{\text {acid }}$ & 0.75 & -0.07 & -0.23 & 0.41 & 0.05 \\
\hline & $\operatorname{SPS} 116 / \varepsilon_{\text {alumina }}^{0}$ & 0.84 & -0.14 & -0.26 & -0.11 & -0.29 \\
\hline & $\mathrm{SPS} 125 / \delta_{\mathrm{H}}$ & 0.70 & -0.14 & -0.15 & -0.09 & -0.08 \\
\hline & Min & 0.31 & -0.44 & -0.49 & -0.42 & -0.46 \\
\hline & Max & 0.87 & $\mathbf{0 . 0 0}$ & -0.08 & 0.52 & 0.41 \\
\hline \multirow[t]{17}{*}{ III } & SPS2 / B & 0.21 & -0.52 & 0.46 & -0.35 & -0.36 \\
\hline & SPS9 / D & -0.05 & -0.45 & 0.31 & -0.29 & -0.46 \\
\hline & $\mathrm{SPS} 12 / \mathrm{E}_{\mathrm{CT}(\pi)}$ & 0.45 & -0.53 & 0.05 & 0.14 & 0.43 \\
\hline & SPS27 / SB & 0.22 & -0.75 & 0.28 & -0.25 & 0.17 \\
\hline & SPS31 / $\beta$ & 0.43 & -0.72 & 0.31 & -0.17 & 0.15 \\
\hline & SPS37 / $\Delta$ & -0.13 & -0.42 & 0.59 & -0.32 & 0.13 \\
\hline & $\operatorname{SPS} 40 / \Delta v_{D}$ & 0.41 & -0.74 & 0.32 & 0.01 & -0.24 \\
\hline & SPS $48 / \Delta v_{\mathrm{CI}}$ & 0.03 & -0.74 & 0.39 & -0.02 & -0.06 \\
\hline & SPS49 / $\Delta v_{\mathrm{OH}}$ & 0.31 & -0.82 & 0.19 & 0.02 & 0.07 \\
\hline & SPS67 / B-2 & 0.18 & -0.66 & 0.51 & -0.25 & -0.12 \\
\hline & SPS68 / $C_{B}$ & -0.17 & -0.55 & 0.55 & -0.13 & -0.27 \\
\hline & SPS69 / D & 0.37 & -0.45 & 0.23 & -0.24 & 0.04 \\
\hline & SPS74 / $\Delta \mathrm{H}_{\text {solv }}^{\mathrm{o}}$ & 0.26 & -0.55 & 0.24 & -0.29 & 0.18 \\
\hline & SPS76 / $-\Delta \mathrm{H}_{\mathrm{BF} 3}^{0}$ & 0.21 & -0.72 & 0.04 & 0.14 & -0.11 \\
\hline & SPS84 / DN & 0.36 & -0.72 & 0.42 & -0.09 & -0.06 \\
\hline & $\mathrm{SPS} 85 / \mathrm{D}_{\pi}$ & 0.12 & -0.65 & -0.16 & 0.01 & 0.30 \\
\hline & $\mathrm{SPS} 108 / \mathrm{Xe}^{\mathrm{R}}$ & -0.02 & -0.87 & -0.14 & -0.07 & -0.14 \\
\hline
\end{tabular}




\begin{tabular}{|c|c|c|c|c|c|c|c|}
\hline & SPS111/ $X_{e}$ & & -0.12 & -0.92 & 0.02 & -0.13 & 0.01 \\
\hline & & Min & -0.17 & -0.92 & -0.16 & -0.35 & -0.46 \\
\hline & & Max & 0.45 & -0.42 & 0.59 & 0.14 & 0.43 \\
\hline \multirow[t]{18}{*}{ IV } & SPS $46 / \pi_{2^{*}}$ & & 0.33 & 0.23 & 0.65 & 0.44 & -0.30 \\
\hline & SPS57 / XX & & -0.18 & 0.09 & 0.67 & 0.53 & -0.18 \\
\hline & SPS71 / PA & & -0.12 & -0.01 & 0.47 & -0.14 & 0.28 \\
\hline & SPS73 / $\Delta \mathrm{H}_{v}$ & & 0.01 & -0.17 & 0.61 & 0.29 & 0.52 \\
\hline & SPS75 $/ \varepsilon^{0}(\mathrm{SVB})$ & & -0.05 & 0.39 & 0.45 & -0.09 & -0.60 \\
\hline & SPS77 / $\mu$ & & 0.33 & 0.03 & 0.54 & -0.04 & -0.55 \\
\hline & SPS83 / X & & 0.10 & 0.46 & 0.64 & 0.14 & -0.40 \\
\hline & SPS86 / Logk & & -0.08 & 0.23 & 0.65 & -0.33 & 0.55 \\
\hline & SPS90 / BB' & & 0.46 & 0.35 & 0.26 & -0.48 & -0.29 \\
\hline & SPS97 / Log L ${ }^{16}$ & & -0.15 & 0.05 & 0.71 & 0.52 & 0.35 \\
\hline & SPS99 / M & & 0.23 & 0.31 & 0.67 & 0.46 & -0.31 \\
\hline & $\operatorname{SPS} 118 / \varepsilon_{\beta}$ & & 0.13 & -0.08 & 0.66 & 0.05 & -0.21 \\
\hline & $\mathrm{SPS} 120 / \mu_{\mathrm{D}}$ & & 0.61 & 0.10 & 0.54 & -0.15 & 0.25 \\
\hline & $\mathrm{SPS} 121 / \pi_{\mathrm{I}}$ & & 0.12 & 0.44 & 0.36 & 0.28 & 0.21 \\
\hline & SPS123 $/ \varepsilon_{\alpha}$ & & 0.28 & 0.75 & 0.39 & 0.06 & -0.17 \\
\hline & SPS127 / P & & 0.29 & 0.35 & 0.57 & 0.39 & -0.32 \\
\hline & & Min & -0.18 & -0.17 & 0.26 & -0.48 & -0.60 \\
\hline & & Max & 0.61 & 0.75 & 0.71 & 0.53 & 0.55 \\
\hline \multirow[t]{19}{*}{ V } & $\mathrm{SPS} 7 / \mathrm{Cu}-\lambda_{\max }$ & & -0.63 & -0.32 & -0.09 & -0.03 & -0.09 \\
\hline & $\mathrm{SPS} 10 / \mathrm{E}_{(\mathrm{NR})}$ & & -0.84 & -0.01 & 0.02 & -0.18 & 0.22 \\
\hline & SPS34 / $\chi_{R}$ & & -0.82 & 0.00 & -0.26 & -0.27 & 0.07 \\
\hline & SPS35 / / & & -0.61 & 0.08 & 0.38 & 0.29 & 0.01 \\
\hline & $\mathrm{SPS} 38 / \Delta \delta \mathrm{CHCl}_{3}$ & & -0.38 & 0.47 & -0.50 & 0.36 & 0.20 \\
\hline & SPS41 / $\theta_{1 \mathrm{~K}}$ & & -0.88 & -0.23 & -0.27 & -0.03 & -0.05 \\
\hline & SPS42 / $\theta_{2 \mathrm{~K}}$ & & -0.48 & 0.30 & 0.62 & 0.21 & -0.30 \\
\hline & SPS43 / $\Lambda$ & & -0.76 & -0.12 & 0.19 & 0.44 & 0.20 \\
\hline & SPS47 / $v_{\mathrm{CE}}$ & & -0.70 & -0.19 & -0.13 & 0.13 & 0.33 \\
\hline & SPS72 / $\Delta_{\text {acid }} \mathrm{H}$ & & -0.58 & 0.09 & 0.16 & -0.47 & 0.12 \\
\hline & SPS93 / DC & & -0.52 & -0.40 & 0.19 & -0.25 & 0.25 \\
\hline & SPS98 / Log P & & -0.61 & 0.05 & 0.31 & 0.52 & 0.11 \\
\hline & $\mathrm{SPS} 106 / \mathrm{V}_{\mathrm{mc}}$ & & -0.38 & -0.24 & 0.53 & 0.38 & 0.56 \\
\hline & SPS109 / $\mathrm{Xn}^{\mathrm{R}}$ & & -0.43 & 0.67 & 0.12 & -0.11 & 0.35 \\
\hline & SPS112 / $X_{n}$ & & -0.52 & 0.62 & -0.01 & -0.35 & 0.13 \\
\hline & SPS $115 /-\Delta \mathrm{H}_{\mathrm{f}}$ & & -0.53 & -0.28 & 0.21 & -0.03 & -0.34 \\
\hline & SPS122 / $\sigma_{1}$ & & -0.38 & -0.13 & 0.59 & 0.45 & 0.47 \\
\hline & & Min & -0.88 & -0.40 & -0.50 & -0.47 & -0.34 \\
\hline & & Max & -0.38 & 0.67 & 0.62 & 0.52 & 0.56 \\
\hline
\end{tabular}

